\title{
Cytokeratin 18 expression pattern correlates with renal cell carcinoma progression: Relationship with Snail
}

\author{
YOSRA MESSAI $^{1,2}$, MUHAMMAD ZAEEM NOMAN $^{1}$, AMINE DEROUICHE $^{3}$, NADIA KOURDA $^{4}$, \\ INTISSAR AKALAY ${ }^{1}$, MERIEM HASMIM ${ }^{1}$, IZABELA STASIK ${ }^{1}$, SARRA BEN JILANI ${ }^{2}$, \\ MOHAMED CHEBIL ${ }^{3}$, ANNE CAIGNARD ${ }^{5}$, BRUNO AZZARONE ${ }^{6}$, ASMA GATI $^{2}$, \\ AMEL BEN AMMAR ELGAAIED ${ }^{2}$ and SALEM CHOUAIB ${ }^{1}$
}

\begin{abstract}
${ }^{1}$ INSERM, U 753, Laboratoire d'Immunologie des Tumeurs Humaines: Interaction effecteurs cytotoxiques-système tumoral, Institut Gustave Roussy Villejuif; ${ }^{2}$ Laboratoire de Génétique, d'Immunologie et de Pathologies Humaines,

Faculté Des Sciences De Tunis; ${ }^{3}$ Service d'Urologie, Hôpital Charles Nicolle de Tunis; ${ }^{4}$ Laboratorie de Cytologie et d'Immunopathologies, Hôpital Charles Nicolle de Tunis; ${ }^{5}$ Institut Cochin, Université Paris Descartes, CNRS (UMR 8104), Inserm U567, Paris; ${ }^{6}$ INSERM, UMR 542, Université de Paris XI, Hôpital Paul Brousse, Villejuif, France
\end{abstract}

Received October 27, 2009; Accepted December 7, 2009

DOI: 10.3892/ijo_00000597

\begin{abstract}
Renal cell carcinoma (RCC) is the most common type of kidney cancer and recent developments in the molecular biology of RCC have identified multiple pathways associated with the development of this cancer. This study aimed at analyzing the expression pattern of cytokeratin 18 (CK18) in RCC patients and its prognostic relevance. We quantified CK18 mRNA expression and protein using real-time reverse transcription quantitative polymerase chain reaction (RTQPCR) and immunohistochemistry, respectively, in paired tumor and non-tumor samples from 42 patients. Our data indicate that CK18 mRNA and proteins levels increased with advanced stage and grade of the disease. Using primary (RCC5) and metastatic renal cell carcinoma (RCC5 met) cell lines, we demonstrated that CK18 expression was 5-fold higher in the metastatic as compared to the primary RCC cell line and correlated with a migratory phenotype characterized by a distinct elongated morphology as revealed by Phalloidin staining. In addition, RCC5 met cells displayed an increased capacity to attach to fibronectin and collagen which was lost following CK18 knock-down. Our data also indicate that the expression of CK18 was associated with increased Snail expression which correlated positively with advanced disease in RCC patients. The present findings suggest that CK18 may play an important role in the progression of RCC and it may be used as a new predictor for RCC.
\end{abstract}

Correspondence to: Dr Salem Chouaib, INSERM U753, Laboratoire d'Immunologie des tumeurs Humaines: Interaction effecteurs cytotoxiques-système tumoral, Institut Gustave Roussy, PR1, 94805 Villejuif Cedex, France

E-mail: chouaib@igr.fr

Key words: renal cell carcinoma, CK18, tumor progression, Snail

\section{Introduction}

Renal cell carcinoma is the most common form of kidney cancer arising from the renal tubule (1). It accounts for $3 \%$ of all adult malignancies and is the most lethal of the common urologic cancers (2). Approximately $20-30 \%$ of patients present with metastatic disease, and $20-40 \%$ of patients undergoing nephrectomy for clinically localized renal cell carcinoma will develop metastases (3). Since renal-cell carcinoma is highly resistant to chemotherapy, interleukin-2 or interferon- $\alpha$ are widely used as first-line treatment of metastatic disease. Although tumor stabilization and regression have been observed with immunotherapy, the clinical benefit of this treatment is insufficient to halt the spread of disease. Response rates with these cytokines are low and response rates with metastatic renal cell carcinoma range from 15 to $30 \%$ (4). Recent advances in the understanding of the pathogenesis, behavior, and molecular biology of metastatic renal cell carcinoma (RCC) have paved the way for developments that may enhance early diagnosis, improve prognostication, and prolong survival $(5,6)$.

In a variety of human malignancies, tumor progression is associated with changes in intermediate filament (IF) expression (7). IFs provide crucial structural support in higher eukaryotic cells and accumulating evidence shows that IFs also participate in various cellular activities including stress responses, cell growth, cell death and cell migration. In epithelial cells, the predominant IF proteins are the keratins, a multigene family of proteins that are divided into type I (K9-K20) and type II (K1-K8) subclasses. Members of the two subclasses form non-covalent hetero-polymers in a 1:1 ratio, and are further assembled into keratin filaments.

The expression of keratins is tissue specific, and is tightly regulated during differentiation. Cytokeratin 8 (CK8) and Cytokeratin 18 (CK18) are the major components of IFs in simple-type epithelial cells, including those in the liver, lung, kidney, pancreas, mammary gland and in tumors that arise 
from these organs. In this regard, CKs are particularly useful tools for the surveillance of carcinomas. Besides their cell structural support and their role in providing mechanical stability to tissues, CKs are implicated in a range of pathological phenotypes seen in patients bearing mutations in epidermal keratins. In addition, evidence has shown that CK18 filaments play an important role in modulating cellular response $(8,9)$.

Snail is a zinc finger transcription factor that triggers the epithelial-mesenchymal transition (EMT) by directly repressing E-cadherin expression and has been implicated in tumor progression $(10,11)$. Recent data have provided evidence that Snail expression is required for metastatic dissemination. Currently, it seems that Snail may play a role in maintenance of the invasive phenotype $(12,13)$. While preliminary information from expression microarray data sets from primary human breast cancers suggests that high levels of Snail are correlated with poor clinical outcome for women with early breast cancer (14), the involvement of Snail in RCC progression and its relationship with CK18 has not yet been established. Although to date, a small number of possible marker genes are known to predict RCC behavior more accurately, a reliable marker for early diagnosis of RCC has not been reported (15). Since RCC is believed to arise from proximal tubule epithelium $(16,17)$ and in order to identify new prognosis markers for clear cell RCC, we have examined in the present study the relationship between CK18 and Snail expression in RCC. Our findings point to the existence of a relationship between CK18 and RCC progression as well as a role for Snail during this process.

\section{Patients and methods}

Patients. The immunohistochemistry study was based on data obtained from the treatment of 40 clear cell carcinomas surgically resected between 2004 and 2008 at the Division of Urology, Charles Nicolle Hospital, Tunisia. Hematoxylin and eosin (H\&E) stained sections for all 40 specimens were reviewed in a blind manner by one pathologist (Table I). Samples for RT-QPCR were obtained from 42 patients with histologically confirmed clear cell subtype RCC, who had not received pre-operative therapy (Table II). Tumors were resected between 2006 and 2009. The staging of tumors was carried out according to the International Union against Cancer (UICC) standards, based on routine imaging methods and laboratory tests. Normal renal tissue $(n=5)$ obtained from kidneys removed because of benign diseases were used to normalize real-time PCR experiments. Appropriate written consent was obtained to use the material in our study.

Cell lines. Primary and metastatic renal cell carcinoma cell lines RCC5 and RCC5 met respectively were obtained from the same patients with conventional RCC as described previously (18). Cells were cultured in DMEM/F12 1:1 medium containing $10 \%$ heat inactivated FCS, $2 \mathrm{mM}$ L-glutamine, $1 \mathrm{mM}$ sodium pyruvate and $1 \%$ Ultroser $\mathrm{G}$ (Gibco BRL, Life Technologies, Cergy Pontoise, France) at $37^{\circ} \mathrm{C}$ in a humidified atmosphere containing $5 \% \mathrm{CO}_{2}$. NK cells were negatively selected from healthy donor peripheral blood mononuclear cells with NK cell isolation kit II (Miltenyi Biotech).
Table I. Summary of clinicopathological findings for RCC patients (immunohistochemistry).

Characteristics

No. of cases $(n=42)$

$\begin{array}{lr}\text { Sex } & \\ \text { Male } & 23 \\ \text { Female } & 19 \\ \text { Metastasis at first diagnosis } & 3 \\ \text { or recurrent metastasis } & \\ \text { Tumor stage } & 17 \\ \text { pT1 } & 17 \\ \text { pT2 } & 8 \\ \text { pT3 } & 0 \\ \text { pT4 } & \end{array}$

Tumor grade

I $\quad 7$

II 13

III 22

IV 0

Table II. Summary of clinicopathological findings for RCC patients (real-time PCR).

\begin{tabular}{lc}
\hline Characteristics & No. of cases $(\mathrm{n}=42)$ \\
\hline Sex & 24 \\
Male & 16 \\
Female & \\
Metastasis at first diagnosis & 1 \\
or recurrent metastasis & \\
Tumor stage & \\
pT1 & \\
pT2 & 21 \\
pT3 & 15 \\
pT4 & 4 \\
Tumor grade & 0 \\
I & \\
II & 12 \\
III & 13 \\
IV & 15 \\
\hline
\end{tabular}

Immunochemistry. Immunohistochemistry was performed on formalin-fixed, paraffin wax embedded samples. Immunostaining was performed with the mouse anti-human CK18 
antibody (1:100, clone DC10, Dako) and the Dako ChemMate Detection kit. Application of the primary antibody was followed by incubation with biotinylated goat anti-mouse immunoglobulin secondary reagent $(1: 100)$ for $35 \mathrm{~min}$. After rinsing in TBS, the slides were then incubated in Dako Duet (K0492) streptavidin-biotin-horseradish peroxidase complex for $35 \mathrm{~min}$, rinsed in TBS and mounted.

The number of CK18-positive cells by immunostaining in each tumor sample was estimated by the same pathologist in a consecutive analysis of all samples on 1 day to ensure maximal internal consistency. The staining results were grouped into normal CK18 expression (100\% stained cells), heterogeneous CK18 expression $(>10 \%$ and $<100 \%$ stained cells), and complete loss of CK18 expression $(<10 \%$ stained cells).

Real-time reverse transcription quantitative polymerase chain reaction ( $R T-Q P C R)$. Total RNA extraction was performed with TRIzol reagents. Tissue expression of CK18 and Snail was analyzed using single-stranded cDNA synthesized from $1 \mu \mathrm{g}$ of total RNA by SuperScript RTII (Life Technologies, Inc.) and a random hexamer primer. Each gene was run in duplicates $\left(40\right.$ cycles at $95^{\circ} \mathrm{C}$ for $15 \mathrm{sec}$ and $60^{\circ} \mathrm{C}$ for $1 \mathrm{~min}$ ) using SYBR Green chemistry on the ABI PRISM 7700 Sequence Detection System (Applied Biosystems, Weiterstadt, Germany). Samples were independently analyzed 2-3 times. Primer sequences (forward and reverse) were as follows: CK18, 5'-GAGCCTGGAGACCGAGAAC-3' and 5'-TTG CGAAGATCTGAGCCC-3'. Snail, 5'-GAGGCGGTGG CAGACTAG-3' and 5'-GACACATCGGTCAGACCAG-3'. Data analysis involved the $\triangle \mathrm{CT}$ method for relative quantification.

Confocal microscopy. Tumor cells were grown on glass coverslips, fixed with $3 \%$ formaldehyde/PBS for $10 \mathrm{~min}$ and permeabilized with $0.1 \%$ Triton X-100/PBS for $5 \mathrm{~min}$, followed by blocking with $1 \%$ bovine serum albumin/PBS for $20 \mathrm{~min}$. The fixed cells were then stained with Alexa Fluor 568-phalloidin (Molecular Probes) for actin staining and with anti-CK18 antibody for CK18 staining followed by Alexa-488 secondary antibody (Molecular Probes). Coverslips were mounted with Vectashield (Vector Laboratories, Burlingame, CA) and analyzed by a Zeiss laser scanning confocal microscope (LSM-510, Jena, Germany). Images were later on analyzed by using LSM Image Examiner software (Zeiss).

Western blot analysis. Western blotting was performed as described earlier (19). Total cellular extracts were prepared by lysing cells in ice-cold buffer containing 1\% NP40, $1 \mathrm{mmol} / \mathrm{l}$ phenylmethylsulfonyl fluoride, $10 \mu \mathrm{g} / \mathrm{ml}$ aprotinin, and $10 \mu \mathrm{g} /$ $\mathrm{ml}$ leupeptin. Equivalent protein extracts (30-50 $\mu \mathrm{g})$ were denatured by boiling in SDS and ß-mercaptoethanol, separated by SDS-PAGE, and transferred onto nitrocellulose membranes (Pierce/Perbio). Blots were then blocked for $1 \mathrm{~h}$ with TBS containing $5 \%$ non-fat dry milk and probed with appropriate antibody for $3 \mathrm{~h}$ with either anti-CK18 (mouse mAb, Dako) or anti-Snail (mouse mAb, Cell Signaling). After washing, blots were incubated with appropriate horseradish peroxidaseconjugated secondary antibody. The complexes were detected using SuperSignal West Pico Chemiluminescent Substrate (Pierce/Perbio).

RNA interference. Gene silencing of CK18 expression was performed by using sequence-specific siRNA, purchased from Qiagen. RNAi experiments were performed as previously reported (19). Briefly, cells were transfected by electroporation with 200 pmol siRNA in a Gene Pulser Xcell Electroporation System (Bio-Rad; $300 \mathrm{~V}, 500 \mu \mathrm{F}$ ) and then allowed to grow for $72 \mathrm{~h}$. Human siRNA target sequences used for CK18 were 5'-GCCGGAUAGUGGAUGGCAA-3' and 5'-GCAUCGUUCUGCAGAUUGA-3'. SiRNA targeting Luciferase, purchased from Sigma-Proligo, was used as a negative control.

Wound healing (migration) assay. RCC5 and RCC5 met were seeded at $1 \times 10^{6}$ in $60-\mathrm{mm}$ diameter culture dishes. Cells were gently scraped with a plastic tip to make wounds. The medium was removed, cells were washed twice with PBS, and serum-free medium was added along with mitomycin-D. The cells were allowed to migrate into the area of wounds for 24 and $48 \mathrm{~h}$. Afterwards, cultures were fixed in $4 \%$ paraformaldehyde and stained with $0.5 \%$ crystal violet. Migration was assessed by taking pictures of three different regions of each wound at 24 and $48 \mathrm{~h}$. Results are representative of three separate experiments.

Adhesion assay. Ninety-six-well plates were coated with type-I collagen $(3 \mu \mathrm{g} / \mathrm{ml})$, type-IV collagen $(3 \mu \mathrm{g} / \mathrm{ml})$, osteopontin $(5 \mu \mathrm{g} / \mathrm{ml})$, or fibronectin $(5 \mu \mathrm{g} / \mathrm{ml})$ overnight at $4^{\circ} \mathrm{C}$ and washed with PBS as described earlier (20). Briefly, cells were detached with PBS-EDTA and seeded in serumfree medium at a concentration of $10^{4}$ cells/well. After $1 \mathrm{~h}$ at $37^{\circ} \mathrm{C}$ wells were gently washed with phosphate-buffered saline, and attached cells were fixed in $4 \%$ paraformaldehyde and stained with $0.5 \%$ crystal violet. Absorbance of each well was read at $620 \mathrm{~nm}$ in a plate reader. Results are expressed as mean value of triplicate determinations.

Cell death analysis. Cells were plated in a 6-well plate at a density of $5 \times 10^{4}$ cells/well and grown overnight. The medium was replaced with either fresh medium (control medium) or with medium supplemented with various concentrations of TRAIL. Early apoptosis was assessed by flow cytometry using Dioc6 and propidium iodide staining analyzed on a FACSCalibur flow cytometer. Data were processed using CellQuest software (Becton-Dickinson) as previously reported (21).

Cytotoxic activity assay. The cytotoxic activity of the NK cells was measured by a conventional $4-\mathrm{h} \mathrm{Cr}^{51}$ release assay (22) by using triplicate cultures in round-bottomed 96-well plates. After $4 \mathrm{~h}$ of co-culture supernatants were transferred to LumaPlate96-wells (Perkin-Elmer), dried down, and counted on a Packard TopCount NXT. Percentage of specific cytotoxicity was calculated conventionally.

Statistical analysis. RT-QPCR results are presented as means \pm SD per group. ANOVA was used to compare groups, followed by multiple comparisons using Bonferroni's post- 
A

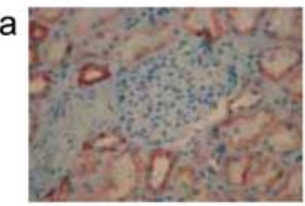

C

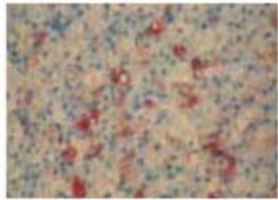

B

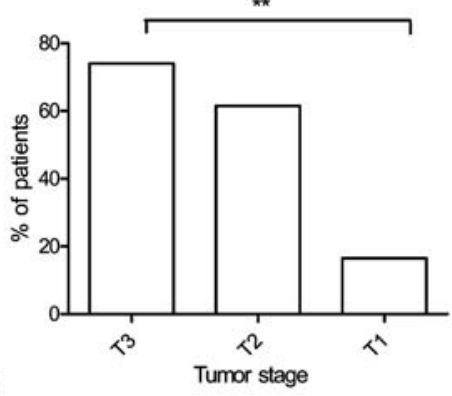

C

a
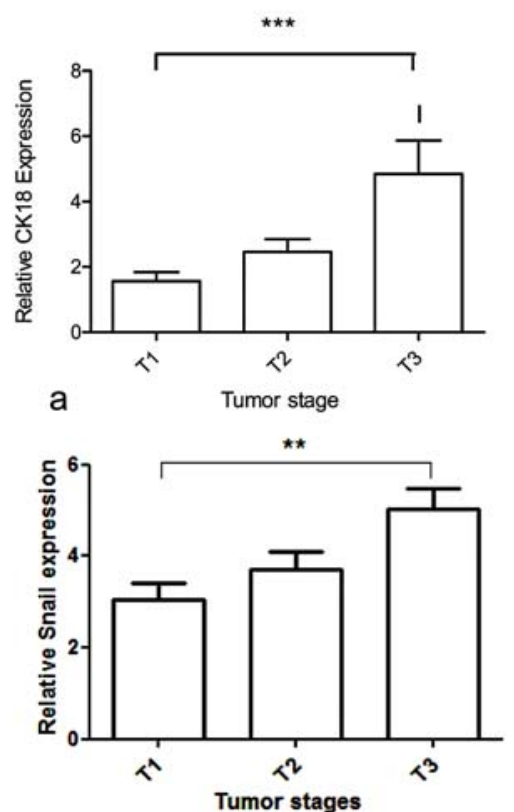
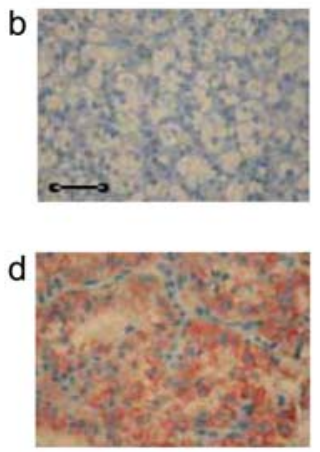

b

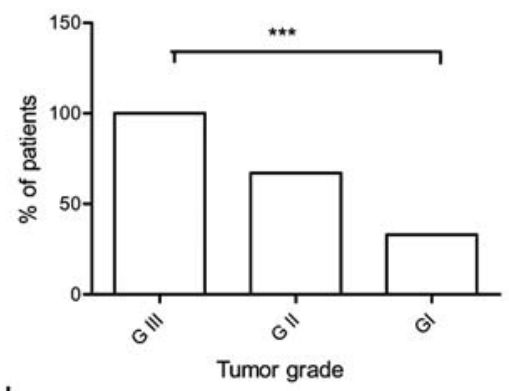

b

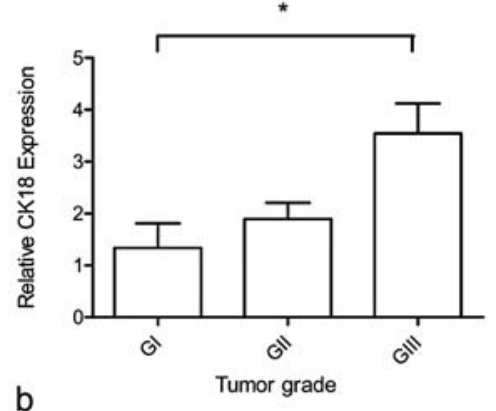

b

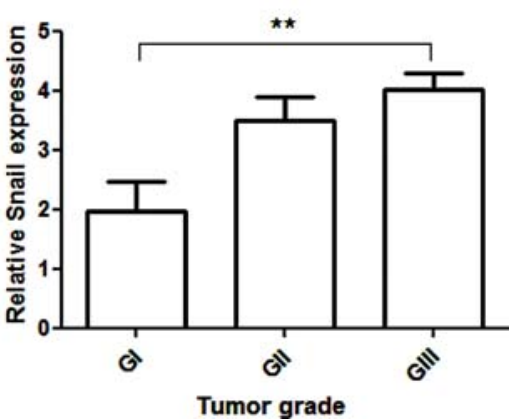

Figure 1. Relationship between CK18, Snail levels and RCC progression. (A) Cytokeratin 18 (CK18) expression levels in renal cell carcinoma (RCC). Immunohistochemical staining of normal kidney and RCC tumors with CK18 monoclonal antibody. Overview of 3 groups of patients according to CK18 expression rate (bar, $25 \mu \mathrm{m}$ ). a, Normal kidney. b, Patients with $<10 \%$ stained tumor cells. c, Patients with 10 to $100 \%(>10 \%$ and $<100 \%)$ stained tumor cells. d, Patients with $100 \%$ stained tumor cells. (B) Relationship between immunohistochemical CK18 expression and clinical features. a, Correlation between CK18 expression and tumor stage: percentage of patients with 100\% stained tumor cells. b, Correlation between CK18 expression and tumor grade: percentage of patients with $100 \%$ stained tumor cells. (C) Relationship between CK18 mRNA expression levels in RCC patients and clinical features. Expression analysis for CK18 was performed by real-time PCR and was normalized to normal renal tissue. a, Correlation between CK18 expression and tumor stage. b, Correlation between CK18 expression and tumor grade. (D) Relationship between Snail expression in RCC patients and clinical features. Expression analysis by RT-QPCR for Snail is relative to 18S rRNA and normalized to normal renal tissue. a, Correlation between Snail expression and tumor stage. b, Correlation between Snail expression and tumor grade.

test and t-test. In all cases, differences were considered significant at: ${ }^{*} \mathrm{P}<0.05 ;{ }^{* *} \mathrm{P}<0.01 ;{ }^{* * *} \mathrm{P}<0.001$.

\section{Results}

The increased expression of CK18 and Snail in RCC patients is associated with advanced tumor stages. CK18 expression was analyzed in RCC tumors from 42 patients by immunochemistry using anti-CK18 mAb on formalin fixed, paraffin wax embedded samples. As shown in Fig. 1A, positive CK18 staining was found in the cell membrane and cytoplasm of all normal tissues of proximal and distal tubules and collecting ducts, while no or weak CK18 expression was observed in the glomerular cells. For RCC patients, three groups were clearly 
A
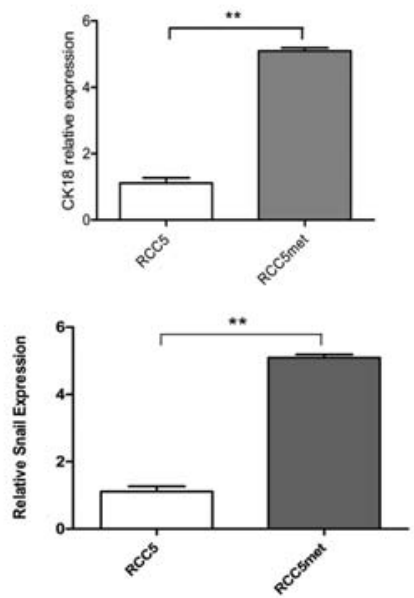

C
B

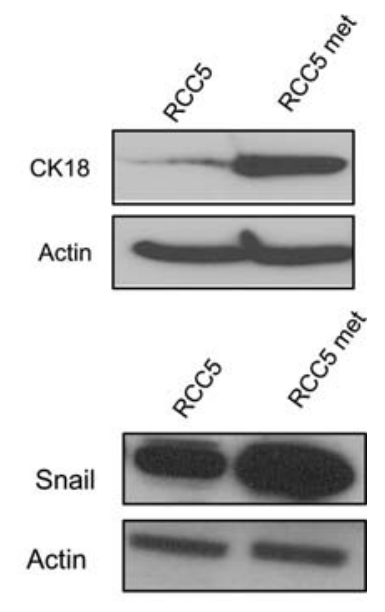

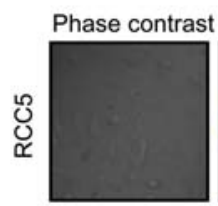
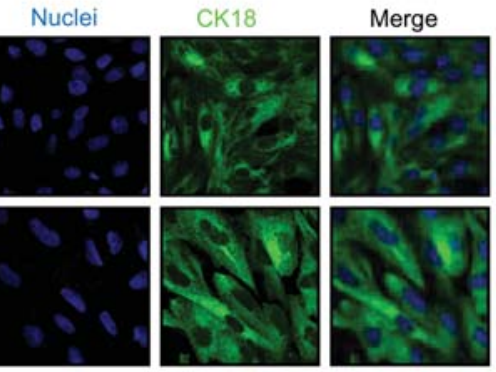

Rainbow
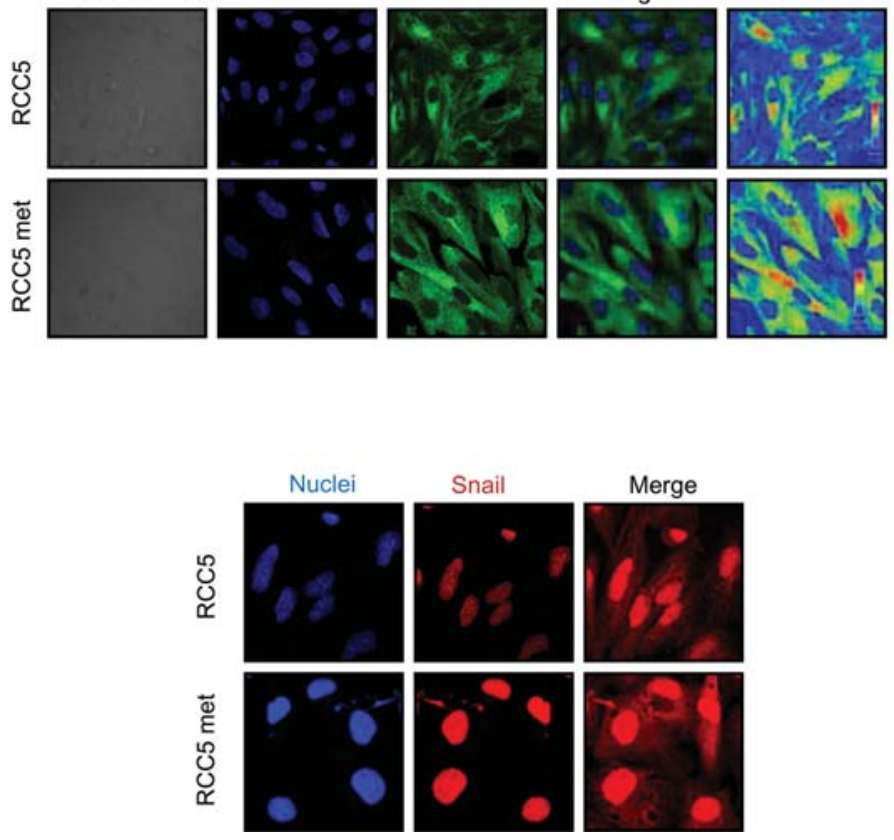

Figure 2. CK18 and Snail levels in primary (RCC5) and metastatic (RCC5 met) cell lines. (A) The expression of CK18 and Snail genes in primary RCC5 and metastatic RCC5 met cell lines was assessed by quantitative RT-PCR. Columns represent mean of three independent experiments; bars, \pm S.D. ${ }^{* *}$ P $\leq 0.01$. Experimental values were normalized to those for the 18S gene. (B) CK18 and Snail expression in RCC5 and RCC5 met cells was determined by Western blot analysis. Actin was used as a loading control. (C) Confocal microscopy analysis of CK18 and Snail protein levels in RCC5 and RCC5 met. Cells were grown on glass coverslips, stained with the appropriate antibody and followed by staining with Alexa-488 secondary antibody. Zeiss Axiovert 200 inverted fluorescence microscope was used for analysis. Data are from one representative experiment of at least three.

distinguishable according to the rate of CK18 expression in tumor cells: patients with $<10 \%$ stained tumor cells (Fig. 1Ab), patients with heterogeneous stained tumor cells $(>10 \%$ and $<100 \%$, Fig. 1Ac) and patients with $100 \%$ stained tumor cells (Fig. 1Ad). Data depicted in Fig. 1B and C (RT-QPCR) indicate that CK18 expression increases according to stage and grade of RCC. We next explored possible correlations between CK18 expression levels and various clinicopathologic characteristics in RCC cases. We evaluated the expression pattern of CK18 by immunohistochemical analysis. We observed that CK18 mRNA levels in human RCC specimens significantly correlate with tumor grade and stage. These results suggest that the CK18 expression pattern could be of predictive value as a marker of disease progression.
In order to evaluate the existence of a correlation between Snail expression and RCC progression, RT-QPCR analysis was performed on patient samples. Similar to what was seen with CK18, we observed that relative Snail expression was higher in RCC patients with either advanced disease stage T3 or advanced disease grade GIII (Fig. 1D).

Differential expression of CK18 and Snail in primary and metastatic RCC cell lines. We investigated CK18 and Snail expression in primary (RCC5) and metastatic (RCC met) RCC cell lines we had previously established from RCC patient biopsies. Using RT-QPCR, data shown in Fig. 2A indicate that the metastatic RCC cell line expressed $\sim 5$-fold higher levels of CK18 mRNA than the primary cells. Similarly the 
A

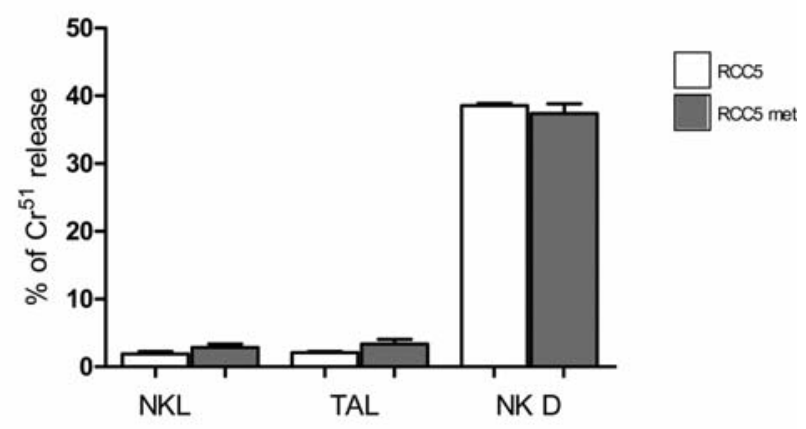

B

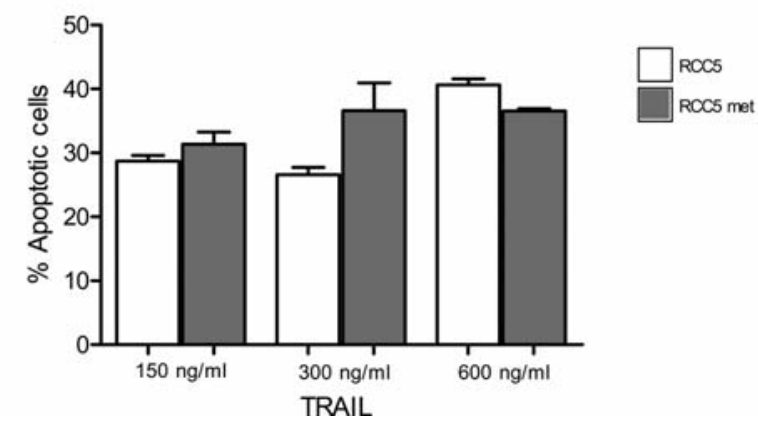

Figure 3. Sensitivity of RCC5 and RCC5 met to NK, TALL and TRAIL mediated lysis. (A) RCC5 and RCC5 met were co-cultured with NK cell line, NK isolated from healthy donors and TALL-104 (ATCC CRL-11386). Cytotoxicity was determined by a conventional 4- $\mathrm{h} \mathrm{Cr}^{51}$-release assay with different effectors at different ratios. Bars, \pm SD. (B) RCC5 and RCC5 met tumor cells were incubated with recombinant Trail $(150,300$ and $600 \mu \mathrm{g} / \mathrm{ml})$ for the indicated times, and cell death was measured by DIOC6 and PI staining.

expression of Snail was higher in RCC5 met as compared to RCC5. Fig. 2B shows the results of Western blot analysis confirming the increased expression of CK18 and Snail proteins in the metastatic RCC cell line. These observations were further confirmed by confocal microscopy analysis (Fig. 2C) showing an over-expression of CK18 and Snail in the metastatic RCC5 cell line.

The increased expression of CK18 does not interfere with RCC cell response to NK-mediated cytotoxicity and TRAIL-induced apoptosis. We asked whether the differential expression of CK18 by RCC 5 and RCC 5 met cell lines correlates with their susceptibility to NK cell- and TRAIL-induced cell death by investigating their cytotoxic response to human NK cells and to TRAIL. Data shown in Fig. 3A clearly indicate that the killing response by cytotoxic effectors (NKL, TALL and peripheral NK) was not affected by the level of CK18 expressed in primary and metastatic targets. In addition, the data depicted in Fig. 3B indicate that RCC5 and RCC5 met display a similar cytotoxic response following treatment with TRAIL suggesting that CK18 does not interfere with the apoptotic machinery in these cells.
Differential adhesive and migratory potential of RCC5 and RCC5 met. Phalloidin staining revealed morphological changes observed in metastatic cells as compared to the primary cell line. In fact, RCC5 met display a migratory phenotype characterized by an elongated morphology, disorganized actin cytoskeleton and the presence of cell extensions associated with a marked increase in filamentous actin cellular content (Fig. 4A). We compared also the adhesive properties of the two RCC cell lines to defined components of the extracellular matrix. We found that metastatic cells RCC5 met attach better to different cell matrices especially fibronectin and collagen I in comparison to the primary cells RCC5 (Fig. 4B).

We next examined the cell motility of RCC5 and RCC5 met on plastic substrate by determining the rate of wound closure after scraping cells from an area of monolayer cultures. Confluent monolayer cells were scraped, and cells were allowed to migrate for 24 and $48 \mathrm{~h}$ in serum deprived medium added with mitomycin-C to inhibit cell proliferation. Primary cell line RCC5 showed an obviously slower closure rate at both 24 and 48 h when compared with metastatic cells RCC5 met (Fig. 4C).

Silencing of CK18 in RCC5 met resulted in the loss of the migratory phenotype. To further assess the putative role of CK18 in migration and adhesion of metastatic cells, transfection of RCC5 met cell line with small interfering RNA (siRNA) targeting CK18 was performed. A specific and efficient reduction of the level of CK18 protein as compared to control siRNA was observed $72 \mathrm{~h}$ after transfection (Fig. 5A). Knockdown of CK18 was accompanied by a decrease in adhesion of metastatic cells to fibronectin and collagen I (Fig. 5B). In addition, as depicted in Fig. 5C, CK18 silencing in RCC5 met resulted in morphological changes showing that RCC5 met acquired shape and monolayer organization similar to that exhibited by RCC5 cells. We further demonstrated that the loss of CK18 in the RCC5 met resulted in the loss of elongated cells (Fig. 5C). All these changes may reflect an attenuation of cell migratory properties.

\section{Discussion}

Renal cell carcinoma (RCC) represents five percent of adult epithelial cancers and clear cell RCC is the most frequent histological subtype. Improved understanding of the molecular pathways implicated in the pathogenesis of RCC has led to the development of specific targeted therapies to treat the disease. In particular, CK18 is attracting considerable interest as a response biomarker during clinical trials of anticancer drugs. Given the important role of CK18 in cell adhesion, cell migration, and signal transduction, which are of major importance in tumor invasion and metastasis, it seemed reasonable to investigate the effect of CK18 expression on patient outcome in renal cell carcinoma. Here we evaluated the expression pattern of CK18 in renal cell carcinoma by immunohistochemical analysis of RCC tumors and studied the correlation between CK18 expression and tumor stage and grade. The comparison of the CK18 expression pattern with different clinicopathological risk factors revealed a highly significant correlation to the size and differentiation grade of the tumor. These parameters are, among other factors, a 
A

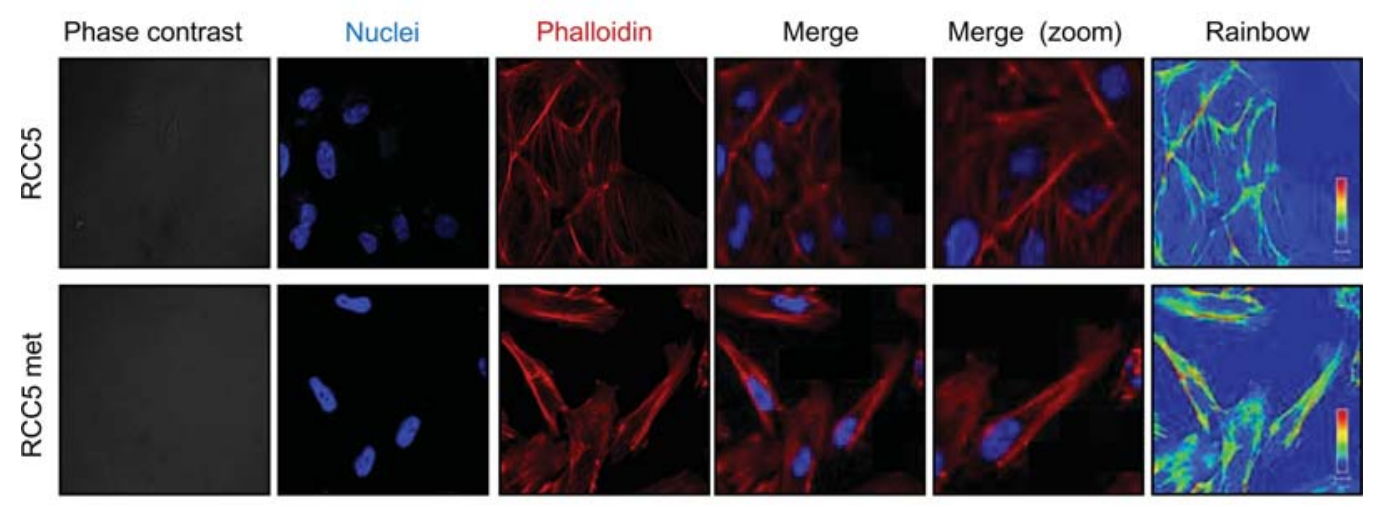

B

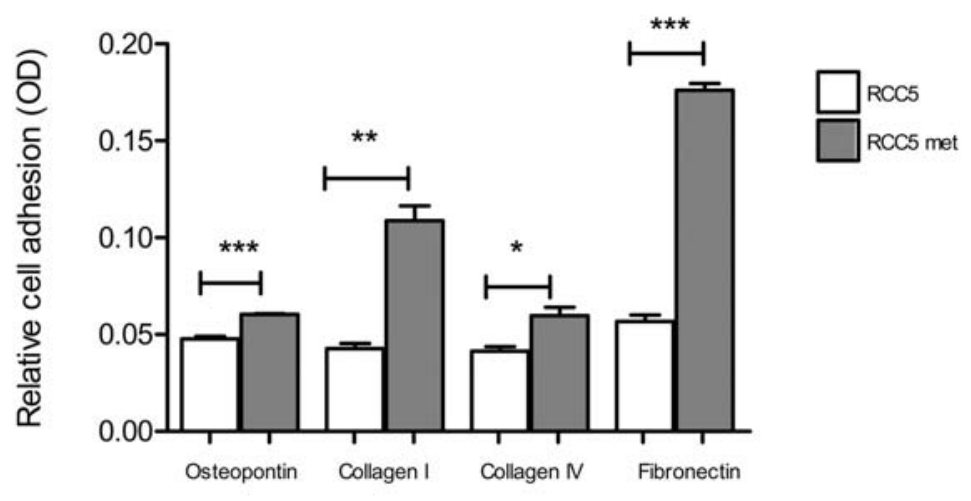

C
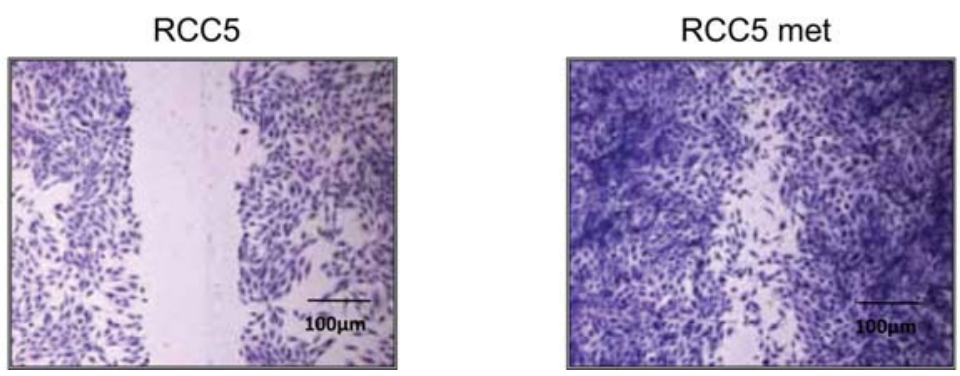

Figure 4. Differential adhesive and migration properties of RCC5 and RCC5 met. (A) RCC5 and RCC5 met were grown on glass coverslips and stained with Rhodamine-phalloidin before analyzing with a Zeiss Axiovert 200 inverted fluorescence microscope. (B) Renal carcinoma cell line adhesion. RCC5 and RCC5 met were tested for their ability to adhere to osteopontin, collagen I, collagen IV and fibronectin. Results are expressed in optical density units (OD) units as a mean of triplicate values \pm SD. (C) Renal carcinoma cell line migration assay. RCC5 and RCC5 met were permitted to migrate into the wound area of monolayer cultures and photomicrographs were taken at $24 \mathrm{~h}$. Results are representative of three separate experiments.

function of the proliferative rate of the primary tumor, suggesting a possible relationship between higher CK18 expression and increased proliferative activity and survival. Moreover, we observed in some early stages and low grade tumors the loss of expression of CK18 as compared with normal renal cells. According to these observations, three hypotheses could be advanced to explain the variation level of CK18 expression in RCC following tumor grades and stages: i) decreased CK18 expression is only needed in low tumor grades and stages to have tumor progression; this expression may be restored in high tumor grade; ii) combination of mutations in different genes may be associated with the weak CK18 expression in low grade phenotype; or iii) the immune system may be effective at low grades of RCC in eliminating CK18 expressing tumor cells but ineffective at high grades. Recently, Walter et al reported a high frequency of CK18 specific CD8 $\mathrm{T}$ cells in healthy donors using an HLA-A2 tetramer refolded with a nonameric peptide isolated from RCC primary tumor $(23,24)$. These potentially autoreactive $\mathrm{T}$ cells may play an essential role in controlling tumor growth.

This suggests an important functional role of this protein in the development or progression of the disease. The variation 
A

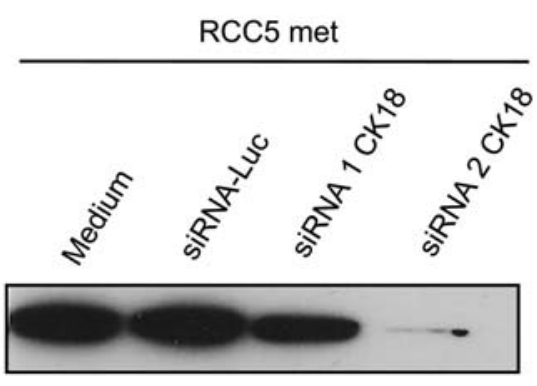

Actin

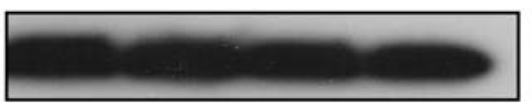

B
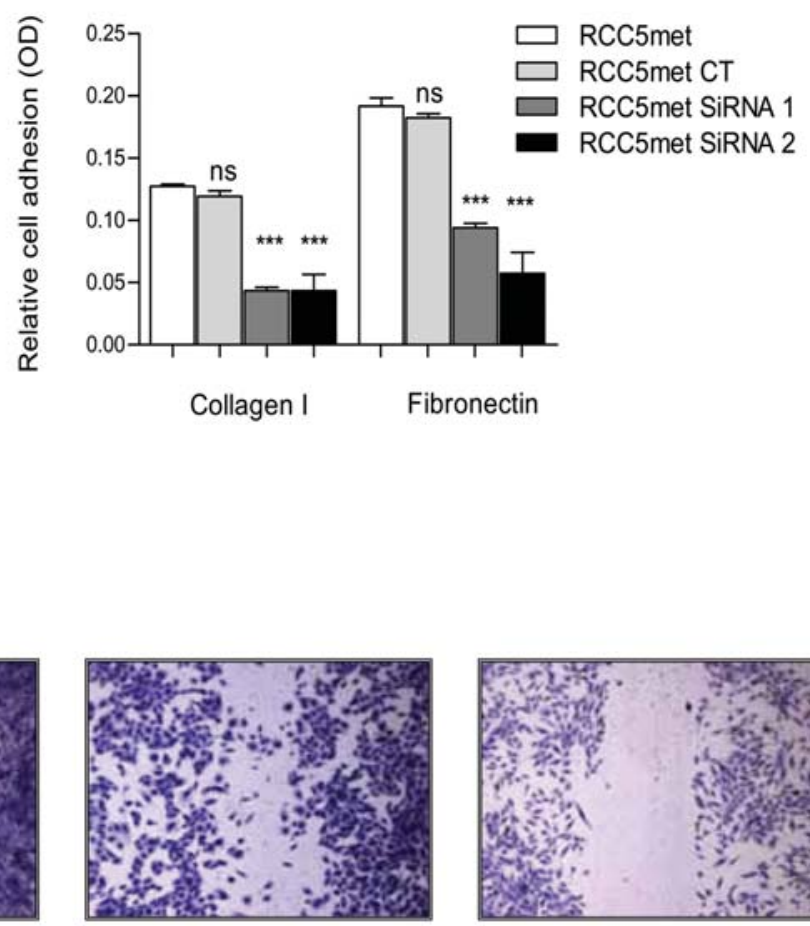

RCC5 met siRNA ctrl

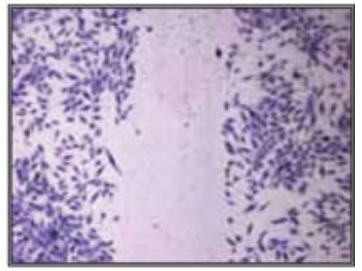

RCC5 met siRNA CK18

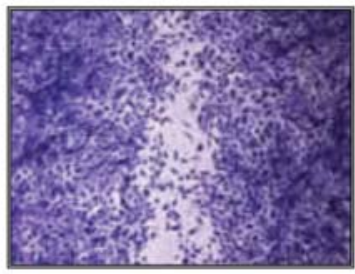

RCC5 met

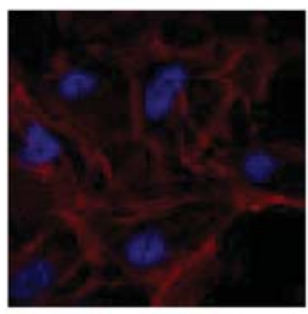

RCC5 met siRNA ctrl

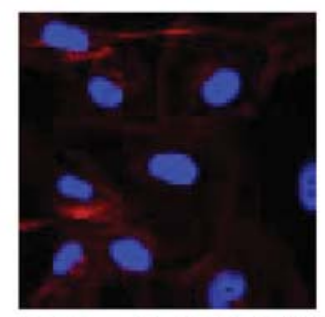

RCC5 met siRNA CK18

Figure 5. Cytokeratin 18 knock-down resulted in the modulation of RCC5 met tumor cell morphology and its adhesive and migratory properties. (A) Knockdown of CK18 down-regulates the protein expression. RCC5 met cells were transfected with either 2 different sequences of CK18 siRNA, or scramble control siRNA (control). Whole-cell lysates were subjected to SDS-PAGE, blotted, and probed with a specific antibody, as indicated. Actin was used as a loading control. (B) RCC5 met transfected with control siRNA (CT) or CK18 siRNA were tested for their ability to adhere to collagen I and fibronectin. Results are expressed in optical density units (OD) units as a mean of triplicate values \pm SD. (C) RCC5 met transfected with control siRNA (CT) or CK18 siRNA were tested for their ability to migrate in a wound healing assay. (D) RNA interference-mediated silencing of CK18 in RCC5 met was accompanied by a change in tumor cell morphology.

of CK18 at protein levels seems to be correlated with mRNA level. The results presented here show that advanced tumor grade and stage of RCC are associated with CK18 mRNA expression. It should be noted that several correlative studies between CK18 expression and prognostic value were previously established with discrepant results depending on the primary tumoral site. In this respect, an association between the induction of CK18 and a poor prognostic outcome has been established in carcinoma of the oral cavity (25) and in non-small cell lung cancer (26). Cintorino et al suggested that CK8, CK18 and CK19 expression and distribution patterns could be of predictive value as markers of disease progression as defined by the appearance of lymph node metastases in oesophageal squamous cell cancer (27).

In this context, one issue was to identify the functional consequences of such expression on tumor behaviour. For this purpose, we used a primary RCC cell line and its metastatic derivative which we have previously established (18). We assessed first using different methods the differential behaviour of the primary and metastatic cell lines including their susceptibility to NK- and Trail-induced cell death and of their adhesion and migration capability. We found that the two cell 
lines have the same response to NK cytotoxicity and Trail induced apoptosis. However, RCC5 met cell line displays morphological changes characteristic of migration phenotype associated with higher adhesion and migration capability as compared to RCC5 primary cell line. This would indicate that, in this case, contrary to immune escape mechanisms, the acquisition of a migratory phenotype is an important feature for tumor progression. It is well established that epithelial cell and tissue architecture is largely determined by cytoskeletal filaments, which are part of an intracellular protein network comprising the cytoskeleton itself, junctional complexes at the cell border, and numerous associated proteins. Previous studies showed that changes in the composition of the cytoskeleton expression result in an increased plasticity, which is required for epithelial tumor cells to become mobile and invasive. Indeed, most cancer cells do not have the physical properties of leukocytes to invade tissues, circulate in the blood, and re-enter new organs. Therefore, epithelial tumor cells need to undergo a fundamental phenotypical change called epithelial-to-mesenchymal transition (EMT) toward more dedifferentiated and malignant states. To investigate the functional consequences of CK18 overexpression and to assess whether its expression patterns could represent putative markers of tumor progression, we analysed CK18 mRNA in both cell lines and found that CK18 was significantly increased in metastatic RCC cell line. Moreover, siRNA targeting CK18 in RCC5 met line resulted in attenuation of cell migratory and adhesive properties. In the present study we clearly indicate that over expression of CK18 correlated with migratory phenotype characterized by a distinct elongated morphology. Cytokeratin 18 appears to interfere with tumor progression without attenuating tumor cell susceptibility to cell or ligand mediated cytotoxicity despite a change in cell morphology. Our findings fit with recent reports indicating that elevated CK expression is associated with invasion and metastasis and points to its involvement in dynamic cell remodelling during cancer progression $(26,28,29)$. They also show that metastatic renal cells display increased cellular motility, which might indicate increased tumor aggressiveness and poor patient prognosis. However, findings from other groups have challenged the value of CK18 as a putative suppressor of tumor progression in breast cancer (30).

Several studies have associated changes in CK expression with an altered cellular behaviour $(31,32)$. In this respect, it has been reported that the overexpression of the CK18 gene in tumorigenic cells could result from a high level of acetylation of histones and/or of factors controlling the activity of the transcription complex (33).

In the course of these studies, we also showed that the expression of the transcription factor Snail was positively correlated with advanced disease in RCC patients. This fits with its increased expression in RCC5 met as compared to primary RCC line. This expression pattern of Snail in patients and cell lines correlate with that of CK18. Taking into account, this observation as well as Snail function and its role as mediator of cell survival, we suggest that Snail could play a role in the regulation of CK18 expression. Preliminary results using siRNA targeting Snail in RCC5 met led to CK18 overexpression (data not shown) in spite of concomitant increase of CK18 and Snail in high grades and stages. A better understanding of the mechanisms associated with CK18 transcription and its involvement in the control of tumor behaviour may therefore allow for the design of improved therapeutic strategies in RCC.

\section{Acknowledgements}

This work was supported by grants from INSERM (Cooperation INSERM/DGRST), the 'Association pour la recherché contre le cancer' (ARC). Yosra Messai is a recipient of a fellowship from INSERM/DGRST.

\section{References}

1. Oosterwijk E, van Muijen GN, Oosterwijk-Wakka JC and Warnaar SO: Expression of intermediate-sized filaments in developing and adult human kidney and in renal cell carcinoma. J Histochem Cytochem 38: 385-392, 1990.

2. Pantuck AJ, Zisman A and Belldegrun A: Biology of renal cell carcinoma: changing concepts in classification and staging. Semin Urol Oncol 19: 72-79, 2001.

3. Janzen NK, Kim HL, Figlin RA and Belldegrun AS: Surveillance after radical or partial nephrectomy for localized renal cell carcinoma and management of recurrent disease. Urol Clin North Am 30: 843-852, 2003.

4. Guida M and Colucci G: Immunotherapy for metastatic renal cell carcinoma: is it a therapeutic option yet? Ann Oncol 18 (Suppl. 6): vi149-152, 2007.

5. Lam JS, Shvarts O and Pantuck AJ: Changing concepts in the surgical management of renal cell carcinoma. Eur Urol 45: 692-705, 2004.

6. Lam JS, Pantuck AJ, Belldegrun AS and Figlin RA: Protein expression profiles in renal cell carcinoma: staging, prognosis, and patient selection for clinical trials. Clin Cancer Res 13: S703-S708, 2007.

7. Moll R, Divo M and Langbein L: The human keratins: biology and pathology. Histochem Cell Biol 129: 705-733, 2008.

8. Linder S: Cytokeratin markers come of age. Tumour Biol 28: 189-195, 2007.

9. Oshima RG, Baribault $\mathrm{H}$ and Caulin $\mathrm{C}$ : Oncogenic regulation and function of keratins 8 and 18. Cancer Metastasis Rev 15: 445-471, 1996.

10. Peinado H, Olmeda D and Cano A: Snail, Zeb and bHLH factors in tumour progression: an alliance against the epithelial phenotype? Nat Rev 7: 415-428, 2007.

11. Cano A, Perez-Moreno MA, Rodrigo I, et al: The transcription factor snail controls epithelial-mesenchymal transitions by repressing E-cadherin expression. Nat Cell Biol 2: 76-83, 2000.

12. Becker KF, Rosivatz E, Blechschmidt K, Kremmer E, Sarbia M and Hofler H: Analysis of the E-cadherin repressor Snail in primary human cancers. Cells Tissues Organs 185: 204-212, 2007.

13. Blanco MJ, Moreno-Bueno G, Sarrio D, et al: Correlation of Snail expression with histological grade and lymph node status in breast carcinomas. Oncogene 21: 3241-3246, 2002.

14. Davidson NE and Sukumar S: Of Snail, mice, and women. Cancer Cell 8: 173-174, 2005.

15. Takahashi M, Yang XJ, Sugimura J, et al: Molecular subclassification of kidney tumors and the discovery of new diagnostic markers. Oncogene 22: 6810-6818, 2003.

16. Oosterwijk E, Ruiter DJ, Wakka JC, et al: Immunohistochemical analysis of monoclonal antibodies to renal antigens. Application in the diagnosis of renal cell carcinoma. Am J Pathol 123: 301-309, 1986.

17. Finstad CL, Cordon-Cardo C, Bander NH, Whitmore WF, Melamed MR and Old LJ: Specificity analysis of mouse monoclonal antibodies defining cell surface antigens of human renal cancer. Proc Natl Acad Sci USA 82: 2955-2959, 1985.

18. Viey E, Fromont G, Escudier B, et al: Phosphostim-activated gamma delta $\mathrm{T}$ cells kill autologous metastatic renal cell carcinoma. J Immunol 174: 1338-1347, 2005.

19. Noman MZ, Buart S, van Pelt J, et al: The cooperative induction of hypoxia-inducible factor-1 alpha and STAT3 during hypoxia induced an impairment of tumor susceptibility to CTL-mediated cell lysis. J Immunol 182: 3510-3521, 2009. 
20. Abouzahr-Rifai S, Hasmim M, Boukerche H, et al: Resistance of tumor cells to cytolytic T lymphocytes involves Rho-GTPases and focal adhesion kinase activation. J Biol Chem 283: 31665-31672, 2008.

21. Hamai A, Meslin F, Benlalam H, et al: ICAM-1 has a critica role in the regulation of metastatic melanoma tumor susceptibility to CTL lysis by interfering with PI3K/AKT pathway. Cancer Res 68: 9854-9864, 2008.

22. Asselin-Paturel C, Megherat S, Vergnon I, et al: Differential effect of high doses versus low doses of interleukin-12 on the adoptive transfer of human specific cytotoxic T lymphocyte in autologous lung tumors engrafted into severe combined immunodeficiency disease-nonobese diabetic mice: relation with interleukin-10 induction. Cancer 91: 113-122, 2001.

23. Walter S, Bioley G, Buhring HJ, et al: High frequencies of functionally impaired cytokeratin 18 -specific $\mathrm{CD} 8^{+} \mathrm{T}$ cells in healthy HLA-A2+ donors. Eur J Immunol 35: 2876-2885, 2005.

24. Weinschenk T, Gouttefangeas C, Schirle M, et al: Integrated functional genomics approach for the design of patient-individual antitumor vaccines. Cancer Res 62: 5818-5827, 2002.

25. Fillies T, Werkmeister R, Packeisen J, et al: Cytokeratin 8/18 expression indicates a poor prognosis in squamous cell carcinomas of the oral cavity. BMC Cancer 6: 10, 2006.

26. Ohgami A, Mitsudomi T, Sugio K, et al: Micrometastatic tumor cells in the bone marrow of patients with non-small cell lung cancer. Ann Thorac Srg 64: 363-367, 1997.
27. Cintorino M, Tripod SA, Santopietro R, et al: Cytokeratin expression patterns as an indicator of tumour progression in oesophageal squamous cell carcinoma. Anticancer Res 21: 4195-4201, 2001.

28. Malzahn K, Mitze M, Thoenes M and Moll R: Biological and prognostic significance of stratified epithelial cytokeratins in infiltrating ductal breast carcinomas. Virchows Arch 433: 119-129, 1998.

29. Kakehashi A, Inoue M, Wei M, Fukushima S and Wanibuchi H: Cytokeratin 8/18 overexpression and complex formation as an indicator of GST-P positive foci transformation into hepatocellular carcinomas. Toxicol Appl Pharmacol 238: 71-79, 2009.

30. Woelfle U, Sauter G, Santjer S, Brakenhoff R and Pantel K: Down-regulated expression of cytokeratin 18 promotes progression of human breast cancer. Clin Cancer Res 10: 2670-2674, 2004.

31. Moll R, Krepler R and Franke WW: Complex cytokeratin polypeptide patterns observed in certain human carcinomas. Differentiation 23: 256-269, 1983.

32. Moll R: Diversity of cytokeratins in carcinomas. Acta Histochem 34: 37-44, 1987.

33. Prochasson P, Gunther M, Laithier M, Fossar N, Lavialle C and Brison O: Transcriptional mechanisms responsible for the overexpression of the keratin 18 gene in cells of a human colon carcinoma cell line. Exp Cell Res 248: 243-259, 1999. 